Article

\title{
Spatially-Explicit Simulation Modeling of Ecological Response to Climate Change: Methodological Considerations in Predicting Shifting Population Dynamics of Infectious Disease Vectors
}

\author{
Radhika Dhingra ${ }^{1}$, Violeta Jimenez ${ }^{1}$, Howard H. Chang ${ }^{2}$, Manoj Gambhir ${ }^{3}$, Joshua S. Fu ${ }^{4}$, \\ Yang Liu ${ }^{1}$ and Justin V. Remais ${ }^{1,5}, *$
}

1 Department of Environmental Health, Rollins School of Public Health, Emory University, 1518 Clifton Rd. NE, Atlanta, GA 30322, USA; E-Mails: rdhingr@emory.edu (R.D.); violeta.jimenez@emory.edu (V.J.); yang.liu@emory.edu (Y.L.)

2 Department of Biostatistics and Bioinformatics, Rollins School of Public Health, Emory University, 1518 Clifton Rd. NE, Atlanta, GA 30322, USA; E-Mail: howard.chang@emory.edu

3 MRC Centre for Outbreak Analysis and Modeling, Department of Infectious Disease Epidemiology, Imperial College London, London, SW7 2AZ, UK; E-Mail: m.gambhir@imperial.ac.uk

4 Department of Civil and Environmental Engineering, University of Tennessee, Knoxville, 62 Perkins Hall, Knoxville, TN 37996, USA; E-Mail: jsfu@utk.edu

5 Program in Population Biology, Ecology and Evolution, Graduate Division of Biological and Biomedical Sciences, Emory University, 1510 Clifton Rd., Atlanta, GA 30322, USA

* Author to whom correspondence should be addressed; E-Mail: justin.remais@emory.edu; Tel.: +1-404-712-8908; Fax: +1-404-727-8744.

Received: 18 May 2013; in revised form: 17 June 2013 / Accepted: 27 June 2013 /

Published: 22 July 2013

\begin{abstract}
Poikilothermic disease vectors can respond to altered climates through spatial changes in both population size and phenology. Quantitative descriptors to characterize, analyze and visualize these dynamic responses are lacking, particularly across large spatial domains. In order to demonstrate the value of a spatially explicit, dynamic modeling approach, we assessed spatial changes in the population dynamics of Ixodes scapularis, the Lyme disease vector, using a temperature-forced population model simulated across a grid of $4 \times 4 \mathrm{~km}$ cells covering the eastern United States, using both modeled (Weather Research and Forecasting (WRF) 3.2.1) baseline/current (2001-2004) and projected (Representative Concentration Pathway (RCP) 4.5 and RCP 8.5; 2057-2059) climate data. Ten dynamic population features (DPFs) were derived from simulated
\end{abstract}


populations and analyzed spatially to characterize the regional population response to current and future climate across the domain. Each DPF under the current climate was assessed for its ability to discriminate observed Lyme disease risk and known vector presence/absence, using data from the US Centers for Disease Control and Prevention. Peak vector population and month of peak vector population were the DPFs that performed best as predictors of current Lyme disease risk. When examined under baseline and projected climate scenarios, the spatial and temporal distributions of DPFs shift and the seasonal cycle of key questing life stages is compressed under some scenarios. Our results demonstrate the utility of spatial characterization, analysis and visualization of dynamic population responses — including altered phenology — of disease vectors to altered climate.

Keywords: vector-borne disease; spatially-explicit; dynamic; population model; Ixodes scapularis; climate change; temperature; population response; deer ticks

\section{Introduction}

Understanding the ecological response to anthropogenic environmental changes, including changes in climate, land use, land cover and other factors, requires quantitative tools to characterize, analyze and visualize dynamic changes in the populations of key organisms of interest. Developing such tools is made difficult by the fact that population responses to environmental change are spatially and temporally complex, particularly for organisms with multiple environmental life stages, such as those that participate in the transmission of vector-borne diseases (VBD). Disease vector populations may exhibit variations in seasonal timing and duration, and their generally non-linear response to environmental signals makes prediction of the risk posed by VBD under altered environmental conditions challenging [1-3]. When exposed to changing climatic conditions, vector distribution and the risks of VBD may shift substantially across time and space $[1,4,5]$. Yet, a great deal of uncertainty remains for many VBD systems [4,6-8], and little is known regarding the dynamic nature of the population response to climate change, particularly vector phenology (timing of life stages), seasonality and the duration of key population events.

While some ecological analyses have characterized the dynamic population response of various plant and arthropod species to external forcings in a spatially explicit fashion (e.g., [9-12]), much analogous work on VBD has neglected the spatial domain [13,14]. Still, other work forgoes system dynamics, instead investigating the spatial patterns of static population measures, such as presence/absence or mean abundance (see, for instance, [15] for Lyme disease and [16] for hantavirus). Such analyses make use of statistical relationships between climate and habitat suitability to estimate, for instance, the potential changes in the distribution of habitat suitability for, or nymphal density of, Ixodes scapularis, the vector of Lyme disease [17,18]. This approach offers little insight into the nature of the population's response over time, such as shifts in peak population timing or variability in population density during key exposure periods (e.g., high season for recreational activities). Given the substantial and continuing disagreement regarding how climate may change the distribution of VBD (e.g., [4,7]), analyses 
capable of assessing the relationship between exogenous forcings and population dynamics in space and time may provide such insights.

What is more, geovisualization of the dynamic VBD response to environmental change could provide key information (e.g., maps summarizing complex spatio-temporal phenomena) for developing policies to respond to shifting risk. Thus, geospatial tools for characterizing, analyzing and predicting the response of VBD to future changes are desirable, and these should emphasize dynamic phenomena known to be important for understanding risk, such as vector phenology and seasonality. Phenology - the timing of life stages - is known to be sensitive to climatic change and is an important determinant of the spatial distribution of arthropods [19,20]. Current models investigating arthropod distribution under future climates generally ignore phenology, instead, establishing a relationship between a vector's current abundance and key habitat characteristics and, then, applying that model to projected future conditions [21,22]. An examination of an organism's phenological response can reveal important, but subtle, impacts of changing climate. For instance, the date of flowering and fruiting have been shown to be important determinants of aspen distribution [23], and the date of first oviposition has been shown to be important for gypsy moth distribution [24]. Characterization of life stage-specific dynamic responses can highlight such subtle determinants of the distribution of vectors under the future climate.

The seasonality of events may also shift under future conditions, with important consequences for VBD risk. For instance, vector populations may peak at certain times of the year, with peak incidence of disease occurring at other times (e.g., see [25] for Lyme disease). Some models of VBD response to climate change attempt to roughly characterize changes in seasonality (e.g., [26]); some integrate seasonal elements, such as temperature, humidity and daytime hours, through degree-day models (e.g., [27-30]), and still, others do not explicitly account for seasonality (e.g., [31]). A more detailed spatial representation of seasonal shifts would make it possible to characterize the potentially profound effect that environmental change may have on the length and timing of VBD transmission seasons.

Here, we develop a spatially-explicit modeling approach for investigating the dynamic population responses of a disease vector of interest, with the goal of enhancing our understanding of future VBD risk. We introduce the concept of dynamic population features (DPFs), which provide information on population cycling, seasonal timing and phenological events across vector life stages. Importantly, we describe how analysis of such features — such as number and timing of population peaks (Table 1) — may be used to predict disease risk.

To demonstrate the utility of this modeling approach, we examine the responses of the black-legged deer tick (Ixodes scapularis), the vector for Lyme disease, to changes in temperature across the eastern United States. I. scapularis is an excellent model organism with which to examine the influence of climate change on phenological and seasonal characteristics: it is known to be highly sensitive to environmental conditions, including temperature $[6,15,25,32]$. Furthermore, the three $I$. scapularis life stages (larva, nymph and adult) require different temperature conditions to support host finding or progress to the next life stage [28]. 
Table 1. Dynamic population features (DPFs) of population response.

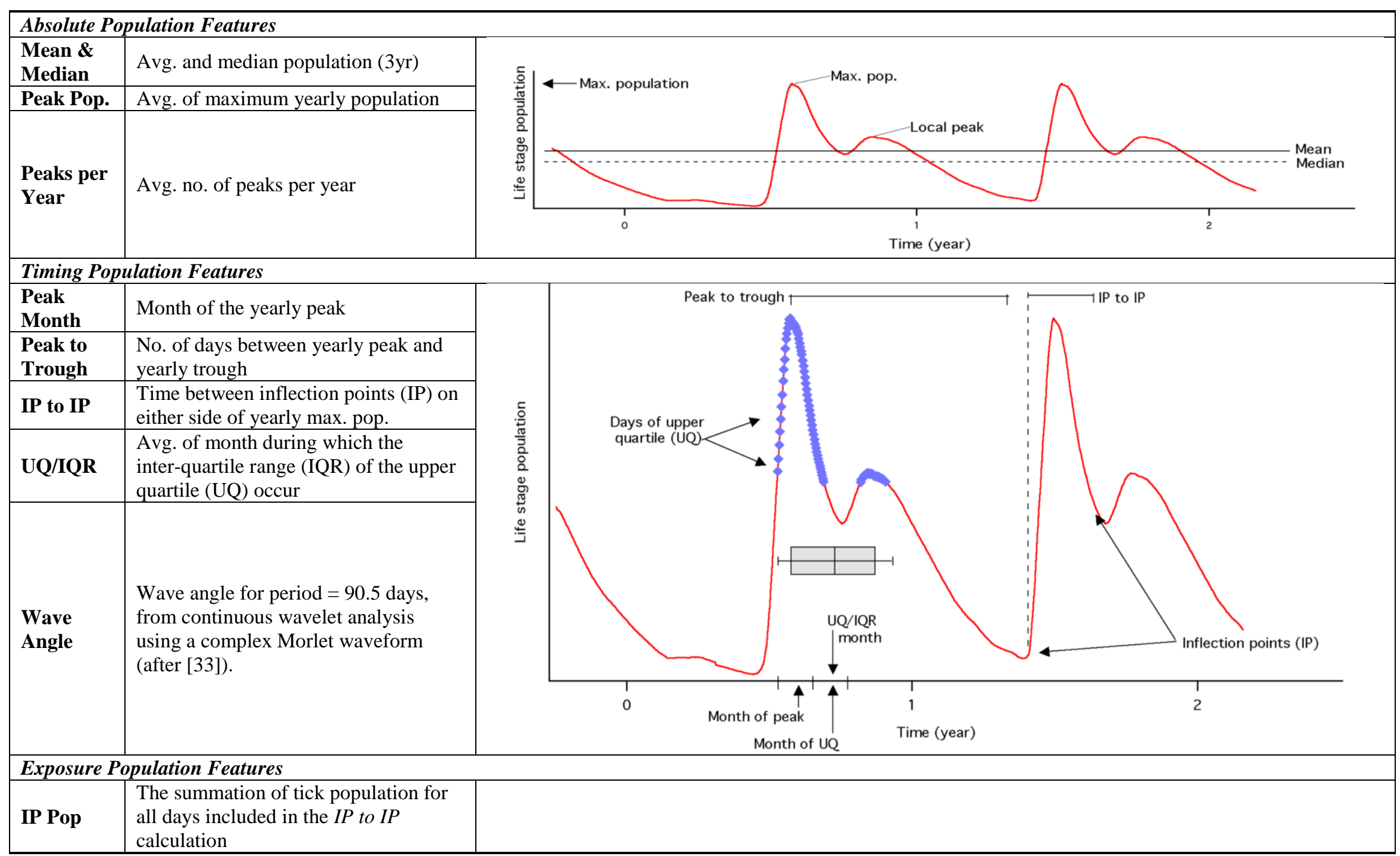


We explore the Lyme disease system as a case study, simulating I. scapularis population dynamics over the eastern United States using modeled climate data, and spatially characterize, analyze and visualize key DPFs for each tick life stage. We examine DPFs from simulated dynamics under current climate conditions and compare these to observed data to ascertain which features best predict current levels of disease risk. We then project DPFs under two future climate scenarios and provide key geovisualizations of projected vector dynamics over the spatial range. We show, by characterizing and visualizing DPFs, how we can determine which population features best predict disease risk under current conditions and can then explore how future conditions may lead to shifts in these same DPFs in the future. We analyze DPFs in the context of I. scapularis and Lyme disease risk, but note that the approach shows promise for other organisms and disease systems.

\section{Methods}

\subsection{Modeling Methodology}

The overarching analysis involved four key steps. First, a deterministic, dynamic population model was run, in parallel, over a large geographic area to generate spatially explicit simulations of population density in response to temperature variation. A daily time step was used in conjunction with the smallest grid cell size for which temperature data were available from a global circulation model. Second, simulated population dynamics were recorded at each grid cell for each vector life stage under current and future climate scenarios, and these were characterized in terms of their dynamic population features (DPFs), which were chosen to highlight population trends, seasonality or a combination of both. Third, DPFs were evaluated for their ability to predict the current distribution of vectors or human disease risk, using publically available data. Finally, DPF values found to be important determinants of current vector or disease distributions were visualized across the spatial domain for a range of future climate scenarios. We describe each of these steps in detail, with the application to Lyme disease, next.

\subsection{Lyme Model}

A twelve-stage temperature-driven life cycle model of black-legged deer ticks (I. scapularis) (described in [28]) was adapted for high-performance simulation using Simulink (v. 7.0) and Matlab (R 2011b) and executed across a cluster of 48 nodes. The model was run using daily (rather than monthly) mean temperatures, and the model was coded to be spatially explicit, executed in parallel at each grid cell across a large spatial domain. Temperature drivers shape simulated tick populations through degree-day functions, which model development delays, and through temperature-dependent activity parameters, which model host-seeking behaviors (see Supplementary Information, Table S1). Using a daily time step, model simulations at each cell were carried out over the domain under both the baseline and projected climate periods. Time series outputs, recorded daily, included questing adult (QA), questing nymph (QN), and questing larva (QL) populations. Given the rather large spatial resolution simulated from the perspective of Ixodes ecology (e.g., [34,35]), populations do not interact between grid cells (i.e., im/emigration are not modeled). 


\subsection{Domain and Climate Data}

Simulation and subsequent analyses were conducted in the eastern United States across a domain of $4 \times 4 \mathrm{~km}$ grid cells. Customized climate data across this grid were obtained from the Regional Climate Model (Weather Research and Forecasting (WRF) 3.2.1) simulated at the University of Tennessee/Oak Ridge National Laboratory [36]. Daily temperatures at each cell were calculated as an average of daily minimum and maximum temperatures produced by climate simulations for a baseline time period (2001-2004) and two projected scenarios of differing severity (for 2057-2059). Projected scenarios, Representative Concentration Pathway (RCP) 4.5 and RCP 8.5, correspond to a continuous rise in radiative forcing to $4.5 \mathrm{~W} / \mathrm{m}^{2}$ (moderate scenario) and $8.5 \mathrm{~W} / \mathrm{m}^{2}$ (severe scenario), respectively, in 2100 [37].

\subsection{Dynamic Features of Ixodes Population Response to Seasonality Shifts}

Dynamic population features, chosen to highlight Ixodes population phenology and seasonality, were determined as described in Table 1 for each year. These were used to compare simulated population dynamics for each life stage at the grid cell level for three years of simulation under both the baseline and projected climate conditions. With the exceptions of the Mean or Median, calculated as the three-year mean or median of the simulated daily population, DPFs were calculated for each year of the simulation period and averaged to produce a final DPF value at each cell for each climate scenario.

Population response DPFs included three-year Mean and three-year Median populations; the maximum population during each year (Peak Population); and the average number of local maxima per year (Peaks per Year). A 90-day moving window was used to identify each local peak through the course of the year. In order to identify peaks in the first 90 days of the simulation output, the last 89 days of simulation output were prepended to the output to provide a 90-day window. Similarly, the first 89 days of simulation output were appended to the simulation in order to aid identification of peaks in the final 90 days of the simulation.

Seasonality DPFs, which were defined for each life stage, included two quantifications of season length and one of season timing. One classification of season length, termed IP to IP, was defined as the number of days between inflection points on either side of the annual maximum population. Inflection points were defined as changes in the concavity of the loess-smoothed (30-day window) population time series; changes in concavity were determined using a 3-point central difference equation on the smoothed time series. The second season length quantification was defined as the number of days from the annual maximum population to the annual minimum population, termed Peak to Trough. Wave Angle may be understood as the relative timing of each cell's season. To determine Wave Angle, continuous wavelet analysis was carried out using a Morlet wavelet [38] for the period of maximum power, 90.5 days (see Supplementary Information). DPFs combining seasonality and absolute population included IP Pop is the number of tick-days during each life stage's season (that is, the summation of the tick population for all days included in the IP to IP calculation). Additionally, $U Q / I Q R$ is used to estimate the period within the calendar year where the highest quartile simulated populations occur. Thus, $U Q / I Q R$ is defined by selecting the time points (days) in which the 
upper quartile populations occur, then taking the mean of the interquartile range of these time points (Table 1).

\subsection{Comparison of DPFs to Observed Data}

DPFs obtained from the model as described above were fit to observed county-level I. scapularis presence (coded in three levels as absent, reported and established) and Lyme disease incidence (coded in four levels as none/minimal, low, medium and high) obtained from the Centers for Disease Control and Prevention (CDC) [33,39]. For all analyses, the four reported classifications were grouped into all possible dichotomizations (e.g., for Lyme disease, dichotomizations included minimal/none vs. low, medium and high; minimal/none and low vs. medium and high; and minimal/none, low and medium $v s$. high). DPFs were spatially averaged to the county level and compared to the observed (CDC) data using both area under the receiver operating characteristic curve (AUC) and logistic regression to ascertain each DPF's predictive ability. AUC (range: 0 to 1) quantizes model predictive accuracy for a dichotomous outcome, where a value of 0.5 indicates no predictive ability, a value of 1 indicates perfect discrimination and a value of 0 indicates lack of discrimination. To assess potential spatial variation in the ability of DPFs to predict Lyme disease risk, AUCs for selected DPFs were also determined for counties in three regions (Northeast, South and Midwest), as defined by the US census.

\subsection{Spatial Sensitivity Analysis}

To assess DPF sensitivity to spatial autocorrelation, the above-defined model outputs were also fit to observed Lyme disease and tick prevalence data using both a (non-spatial) logistic model and a spatial logistic model. The spatial regression model is defined as follows:

$$
\operatorname{logit}\left[P\left(y_{i}=1\right)\right]=\alpha+\lambda_{i}+\beta X_{i}
$$

where $y_{i}$ is the dichotomized observed Lyme disease category, $\alpha$ is the overall baseline risk, $\lambda_{i}$ is the county-specific spatial random effects and $\beta$ represents the log odds ratio associated with DPFs of population response $\left(X_{i}\right)$. The model used controls for the effects of spatial autocorrelation using an intrinsic conditional autoregressive (CAR) model [40]. The CAR model, often formulated by the conditional distribution of $\lambda_{i}$, given its neighbors, assumes that $\lambda_{i}$, for each county, $i$, is a spatial average of its neighbors. The conditional distribution is Gaussian, with mean $\frac{1}{m_{i}} \sum_{i \sim i^{\prime}} \lambda_{i}$ and variance $\tau^{2} / m_{i}$, where $i \sim i^{\prime}$ denotes that county, $i$, shares a boundary with county $i^{\prime}$, and $m_{i}$ is the number of boundary-sharing neighbors for county $i$. The percent change in $\beta$ and the magnitude of parameter, $\tau^{2}$, which controls the degree of spatial similarly, were used to assess the improvement in fit provided by the addition of the spatial term to the logistic regression model.

\section{Results}

Simulated population dynamics for the three questing tick life stages were obtained from a temperature-forced model simulated across a grid of $4 \times 4 \mathrm{~km}$ cells covering the eastern United States under both baseline and projected climate. Based on these simulated population dynamics, ten dynamic population features (DPFs) were derived and analyzed spatially to characterize the population 
response to current and future climate across the domain. Pairwise correlations between DPFs were determined, and each DPF under the current climate was assessed for its ability to discriminate Lyme disease risk and vector presence/absence using observed data from the US CDC.

\subsection{Correlation among DPFs}

To determine the degree to which DPFs are collinear and, thus, to quantify related aspects of the population curve, correlations were calculated for all pairs of DPFs. There was strong correlation between different DPFs of absolute population (Mean, Median, and Peak Population) in all questing life stages over the spatial extent of analysis $\left(\mathrm{r}_{\mathrm{s}} \geq 0.96\right.$; see Table 2). Peaks per Year and IP Pop were also highly correlated $\left(\mathrm{r}_{\mathrm{s}} \geq 0.88\right)$ with absolute population DPFs and each other in the questing adult stage, while Peaks per Year was also highly correlated $\left(\mathrm{r}_{\mathrm{s}} \geq 0.90\right)$, with absolute population DPFs and $I P$ to IP in the questing nymph and larval stages. Only Wave Angle was inversely correlated with all other DPFs in the nymph and adult stages. With the exception of its correlation with $U Q / I Q R\left(\mathrm{QA}: \mathrm{r}_{\mathrm{s}}=-0.77\right.$; QN: $\left.\mathrm{r}_{\mathrm{s}}=-0.81\right)$, this inverse relationship was weak $\left(\mathrm{r}_{\mathrm{s}} \geq-0.63\right)$. In the questing larval stage, only IP to $I P$ showed a weak inverse correlation with other DPFs (save for its correlation with $U Q / I Q R$ ). No timing DPF was strongly associated with any other timing DPF in any life stage.

Table 2. Spearman correlation coefficients $\left(\mathrm{r}_{\mathrm{s}}\right) *$ were assessed between DPFs at each cell for each questing life stage under the baseline climate scenario.

\begin{tabular}{|c|c|c|c|c|c|c|c|c|c|c|}
\hline & Mean & Median & $\begin{array}{c}\text { Peak } \\
\text { Population }\end{array}$ & $\begin{array}{l}\text { Peaks per } \\
\text { Year }\end{array}$ & $\begin{array}{c}\text { Peak } \\
\text { Month }\end{array}$ & $\begin{array}{l}\text { Peak to } \\
\text { Trough }\end{array}$ & $\begin{array}{l}\text { IP to } \\
\text { IP }\end{array}$ & $\begin{array}{c}\text { IP } \\
\text { Pop }\end{array}$ & UQ/IQR & $\begin{array}{l}\text { Wave } \\
\text { Angle }\end{array}$ \\
\hline \multicolumn{11}{|l|}{ Questing Adult } \\
\hline Mean & 1 & 0.98 & -0.48 & 0.99 & -0.47 & 0.25 & 0.26 & 0.98 & 0.55 & -0.34 \\
\hline Median & 0.98 & 1 & -0.47 & 0.96 & -0.46 & 0.24 & 0.19 & 0.94 & 0.47 & -0.24 \\
\hline $\begin{array}{c}\text { Peak } \\
\text { Population }\end{array}$ & -0.48 & -0.47 & 1 & -0.46 & 0.86 & 0.11 & -0.17 & -0.48 & -0.11 & 0.10 \\
\hline $\begin{array}{c}\text { Peaks per } \\
\text { Year }\end{array}$ & 0.99 & 0.96 & -0.46 & 1 & -0.44 & 0.26 & 0.29 & 0.99 & 0.60 & -0.38 \\
\hline Peak Month & -0.47 & -0.46 & 0.86 & -0.44 & 1 & 0.08 & -0.19 & -0.47 & -0.14 & 0.08 \\
\hline $\begin{array}{l}\text { Peak to } \\
\text { Trough }\end{array}$ & 0.25 & 0.24 & 0.11 & 0.26 & 0.08 & 1 & 0.09 & 0.23 & 0.47 & -0.33 \\
\hline IP to IP & 0.26 & 0.19 & -0.17 & 0.29 & -0.19 & 0.09 & 1 & 0.40 & 0.48 & -0.38 \\
\hline IP Pop & 0.98 & 0.94 & -0.48 & 0.99 & -0.47 & 0.23 & 0.40 & 1 & 0.63 & -0.41 \\
\hline UQ/IQR & 0.55 & 0.47 & -0.11 & 0.60 & -0.14 & 0.47 & 0.48 & 0.63 & 1 & -0.77 \\
\hline Wave Angle & -0.34 & -0.24 & 0.10 & -0.38 & 0.08 & -0.33 & -0.38 & -0.41 & -0.77 & 1 \\
\hline \multicolumn{11}{|c|}{ Questing Nymphs } \\
\hline Mean & 1 & 1.00 & -0.42 & 0.99 & 0.20 & 0.17 & 0.99 & 0.37 & 0.60 & -0.45 \\
\hline Median & 1.00 & 1 & -0.41 & 0.98 & 0.21 & 0.18 & 0.98 & 0.38 & 0.62 & -0.47 \\
\hline $\begin{array}{c}\text { Peak } \\
\text { Population }\end{array}$ & -0.42 & -0.41 & 1 & -0.43 & -0.59 & -0.01 & -0.43 & -0.11 & -0.10 & 0.02 \\
\hline $\begin{array}{c}\text { Peaks per } \\
\text { Year }\end{array}$ & 0.99 & 0.98 & -0.43 & 1 & 0.19 & 0.14 & 0.99 & 0.35 & 0.55 & -0.40 \\
\hline Peak Month & 0.20 & 0.21 & -0.59 & 0.19 & 1 & -0.25 & 0.17 & 0.12 & 0.03 & 0.17 \\
\hline IP to IP & 0.17 & 0.18 & -0.01 & 0.14 & -0.25 & 1 & 0.26 & 0.49 & 0.58 & -0.63 \\
\hline IP Pop & 0.99 & 0.98 & -0.43 & 0.99 & 0.17 & 0.26 & 1 & 0.40 & 0.61 & -0.46 \\
\hline $\begin{array}{l}\text { Peak to } \\
\text { Trough }\end{array}$ & 0.37 & 0.38 & -0.11 & 0.35 & 0.12 & 0.49 & 0.40 & 1 & 0.71 & -0.51 \\
\hline UQ/IQR & 0.60 & 0.62 & -0.10 & 0.55 & 0.03 & 0.58 & 0.61 & 0.71 & 1 & -0.82 \\
\hline Wave Angle & -0.45 & -0.47 & 0.02 & -0.40 & 0.17 & -0.63 & -0.46 & -0.51 & -0.82 & 1 \\
\hline
\end{tabular}


Table 2. Cont.

\begin{tabular}{|c|c|c|c|c|c|c|c|c|c|c|}
\hline & Mean & Median & $\begin{array}{c}\text { Peak } \\
\text { Population }\end{array}$ & $\begin{array}{c}\text { Peaks per } \\
\text { Year }\end{array}$ & $\begin{array}{c}\text { Peak } \\
\text { Month }\end{array}$ & $\begin{array}{l}\text { Peak to } \\
\text { Trough }\end{array}$ & $\begin{array}{c}\text { IP to } \\
\text { IP }\end{array}$ & $\begin{array}{c}\text { IP } \\
\text { Pop }\end{array}$ & UQ/IQR & $\begin{array}{l}\text { Wave } \\
\text { Angle }\end{array}$ \\
\hline \multicolumn{11}{|c|}{ Questing Larvae } \\
\hline Mean & 1 & 0.96 & -0.43 & 0.98 & 0.20 & -0.52 & 0.97 & 0.50 & 0.55 & 0.36 \\
\hline Median & 0.96 & 1 & -0.37 & 0.90 & 0.17 & -0.65 & 0.88 & 0.44 & 0.70 & 0.27 \\
\hline $\begin{array}{c}\text { Peak } \\
\text { Population }\end{array}$ & -0.43 & -0.37 & 1 & -0.43 & -0.57 & 0.17 & -0.43 & 0.03 & -0.09 & 0.13 \\
\hline $\begin{array}{c}\text { Peaks per } \\
\text { Year }\end{array}$ & 0.98 & 0.90 & -0.43 & 1 & 0.20 & -0.44 & 0.99 & 0.55 & 0.46 & 0.45 \\
\hline Peak Month & 0.20 & 0.17 & -0.57 & 0.20 & 1 & -0.11 & 0.19 & -0.03 & 0.00 & -0.06 \\
\hline IP to IP & -0.52 & -0.65 & 0.17 & -0.44 & -0.11 & 1 & -0.39 & -0.38 & -0.92 & -0.16 \\
\hline IP Pop & 0.97 & 0.88 & -0.43 & 0.99 & 0.19 & -0.39 & 1 & 0.55 & 0.42 & 0.46 \\
\hline $\begin{array}{l}\text { Peak to } \\
\text { Trough }\end{array}$ & 0.50 & 0.44 & 0.03 & 0.55 & -0.03 & -0.38 & 0.55 & 1 & 0.41 & 0.74 \\
\hline UQ/IQR & 0.55 & 0.70 & -0.09 & 0.46 & 0.00 & -0.92 & 0.42 & 0.41 & 1 & 0.17 \\
\hline Wave Angle & 0.36 & 0.27 & 0.13 & 0.45 & -0.06 & -0.16 & 0.46 & 0.74 & 0.17 & 1 \\
\hline
\end{tabular}

$*$ All values are significant at $p<0.0005$.

\subsection{Comparison of DPFs to Observed Data}

Each DPF was fit to observed tick presence or to Lyme disease risk from the US CDC, producing an AUC value, a discriminatory index that allows comparison of continuous predictions to dichotomous observations without requiring subjective cut points. Peak Month and Peak Population showed the greatest discriminatory ability across all life stages when compared with CDC Lyme disease risk data (Table 3; AUC $=0.54$ to 0.90). Among most DPFs, the minimal vs. high or the minimal/low/medium vs. high dichotomizations gave higher AUC values than the remaining dichotomizations. The dichotomization of minimal vs. low/medium/high performed worst in AUC analyses across all questing life stages. Peak to Trough, IP to IP, IP Pop, UQ/IQR, and Wave Angle showed inconsistent predictive ability over all three life stages. Of these, only IP Pop in the QN life stage showed some improved discriminatory ability.

Comparison of DPFs to CDC tick presence data showed markedly less discriminatory ability. Peak Population, Peak Month, Peak to Trough and UQ/IQR were statistically significant predictors of tick presence across all questing life stages and dichotomizations of CDC data, but AUC values were low and ranged from 0.53 to 0.71 for QA, from 0.54 to 0.69 for $\mathrm{QN}$, and from 0.52 to 0.69 for QL. For QL Wave Angle and tick presence, AUC ranged from 0.66 to 0.7. Along with QA Peak Month and QA Peak Population, this was among the strongest predictors of tick presence. However, for the dichotomization minimal/none vs. low/medium/high, DPFs, Mean, Median, Peaks per Year, IP to IP, $I P$ Pop and $U Q / I Q R$, were uniformly non-significant across all questing life stages. Peak Month AUC values were very similar for QN and QL, and slightly lower for QA. 
Table 3. Area under the receiver operating characteristic curve (AUC) analysis comparing dynamic population features (DPFs) to observed Lyme disease incidence and tick presence.

\begin{tabular}{|c|c|c|c|c|c|c|c|c|c|c|c|}
\hline $\begin{array}{c}\text { Observational Data Set / } \\
\text { Dichotomization }\end{array}$ & $\mathbf{N}$ & Mean & Median & $\begin{array}{c}\text { Peak } \\
\text { Population }\end{array}$ & $\begin{array}{l}\text { Number } \\
\text { Peaks/Yr }\end{array}$ & $\begin{array}{c}\text { Peak } \\
\text { Month }\end{array}$ & $\begin{array}{l}\text { Peak to } \\
\text { Trough }\end{array}$ & $\begin{array}{l}\text { IP to } \\
\text { IP }\end{array}$ & $\begin{array}{c}\text { IP } \\
\text { Pop }\end{array}$ & $\begin{array}{l}\text { UQ/ } \\
\text { IQR }\end{array}$ & $\begin{array}{l}\text { Wave } \\
\text { Angle }\end{array}$ \\
\hline \multicolumn{12}{|l|}{ Questing Adults } \\
\hline \multicolumn{12}{|l|}{ Lyme disease risk } \\
\hline Minimal $v s$. Low/Medium/High & 1,683 & 0.47 & 0.48 & 0.73 & 0.45 & 0.70 & 0.60 & 0.56 & 0.45 & 0.62 & 0.51 \\
\hline Minimal/Low vs. Medium/High & 1,683 & 0.64 & 0.67 & 0.81 & 0.62 & 0.82 & 0.53 & 0.48 & 0.60 & 0.58 & 0.59 \\
\hline Minimal/Low/Medium vs. High & 1,683 & 0.72 & 0.74 & 0.83 & 0.72 & 0.84 & 0.74 & 0.63 & 0.71 & 0.68 & 0.61 \\
\hline Minimal vs. High & 844 & 0.71 & 0.73 & 0.90 & 0.70 & 0.89 & 0.69 & 0.61 & 0.68 & 0.63 & 0.60 \\
\hline \multicolumn{12}{|l|}{ Tick presence } \\
\hline None vs. Reported/Established & 1,683 & 0.52 & 0.52 & 0.69 & 0.54 & 0.67 & 0.60 & 0.54 & 0.54 & 0.61 & 0.53 \\
\hline None/Reported vs. Established & 1,683 & 0.48 & 0.49 & 0.67 & 0.47 & 0.67 & 0.53 & 0.52 & 0.46 & 0.57 & 0.52 \\
\hline None vs. Established & 1,305 & 0.47 & 0.49 & 0.71 & 0.46 & 0.70 & 0.56 & 0.53 & 0.45 & 0.60 & 0.52 \\
\hline \multicolumn{12}{|l|}{ Questing Nymphs } \\
\hline \multicolumn{12}{|l|}{ Lyme disease risk } \\
\hline Minimal vs. Low/Medium/High & 1,683 & 0.46 & 0.45 & 0.70 & 0.46 & 0.54 & 0.57 & 0.53 & 0.47 & 0.60 & 0.56 \\
\hline Minimal/Low vs. Medium/High & 1,683 & 0.70 & 0.70 & 0.79 & 0.68 & 0.76 & 0.61 & 0.71 & 0.71 & 0.76 & 0.79 \\
\hline Minimal/Low/Medium vs. High & 1,683 & 0.75 & 0.74 & 0.80 & 0.75 & 0.90 & 0.40 & 0.56 & 0.75 & 0.61 & 0.69 \\
\hline Minimal vs. High & 844 & 0.73 & 0.73 & 0.86 & 0.73 & 0.92 & 0.65 & 0.56 & 0.74 & 0.55 & 0.67 \\
\hline \multicolumn{12}{|l|}{ Tick presence } \\
\hline None vs. Reported/Established & 1,683 & 0.53 & 0.53 & 0.67 & 0.53 & 0.55 & 0.54 & 0.54 & 0.52 & 0.55 & 0.51 \\
\hline None/Reported vs. Established & 1,683 & 0.49 & 0.49 & 0.65 & 0.49 & 0.69 & 0.58 & 0.54 & 0.50 & 0.53 & 0.48 \\
\hline None $v s$. Established & 1,305 & 0.48 & 0.52 & 0.69 & 0.48 & 0.68 & 0.58 & 0.55 & 0.49 & 0.54 & 0.49 \\
\hline \multicolumn{12}{|l|}{ Questing Larvae } \\
\hline \multicolumn{12}{|l|}{ Lyme disease risk } \\
\hline Minimal vs. Low/Medium/High & 1,683 & 0.46 & 0.54 & 0.70 & 0.45 & 0.54 & 0.73 & 0.58 & 0.46 & 0.58 & 0.75 \\
\hline Minimal/Low vs. Medium/High & 1,683 & 0.69 & 0.73 & 0.79 & 0.66 & 0.76 & 0.52 & 0.75 & 0.65 & 0.78 & 0.46 \\
\hline Minimal/Low/Medium vs. High & 1,683 & 0.74 & 0.72 & 0.80 & 0.75 & 0.90 & 0.52 & 0.62 & 0.73 & 0.63 & 0.58 \\
\hline Minimal vs. High & 844 & 0.73 & 0.71 & 0.85 & 0.72 & 0.92 & 0.37 & 0.58 & 0.71 & 0.61 & 0.58 \\
\hline \multicolumn{12}{|l|}{ Tick presence } \\
\hline None vs. Reported/Established & 1,683 & 0.53 & 0.53 & 0.68 & 0.54 & 0.55 & 0.66 & 0.55 & 0.53 & 0.54 & 0.70 \\
\hline None/Reported vs. Established & 1,683 & 0.49 & 0.49 & 0.65 & 0.48 & 0.69 & 0.65 & 0.53 & 0.48 & 0.52 & 0.66 \\
\hline None $v s$. Established & 1,305 & 0.52 & 0.52 & 0.69 & 0.47 & 0.68 & 0.69 & 0.55 & 0.47 & 0.53 & 0.71 \\
\hline
\end{tabular}

* Bold indicates significance; orange indicates AUC $>0.8$ and $p<0.05$; blue indicates $0.8>$ AUC $>0.7$ and $p<0.05$. 


\subsubsection{Regional Analyses}

A regional analysis, analogous to the preceding AUC analysis, was carried out for Peak Month and Peak Population, to ascertain the degree to which discriminatory ability varies by location. In most cases, AUCs for Peak Month and Peak Population were observed to be highest (Table 4) in the Midwest region of the eastern United States (AUC: 0.80 to 0.96), where the AUC was most often statistically significant. AUC values in the North were consistently lower than the Midwest (AUC: 0.71 to 0.78 ), for both DPFs. In the South, Peak Month usually demonstrated higher statistically significant predictive ability for QN and QL than Peak Population, while both DPFs demonstrated similar predictive ability for QA.

Table 4. Regional AUC * sub-analyses.

\begin{tabular}{|c|c|c|c|c|c|c|c|c|c|}
\hline \multirow[b]{2}{*}{$\begin{array}{l}\text { Observational Data } \\
\text { Set/Dichotomization }\end{array}$} & \multicolumn{3}{|c|}{ Midwest } & \multicolumn{3}{|c|}{ North } & \multicolumn{3}{|c|}{ South } \\
\hline & $\mathbf{N}$ & $\begin{array}{c}\text { Peak } \\
\text { Month }\end{array}$ & $\begin{array}{c}\text { Peak } \\
\text { Population } \\
\end{array}$ & $\mathbf{N}$ & $\begin{array}{c}\text { Peak } \\
\text { Month }\end{array}$ & $\begin{array}{c}\text { Peak } \\
\text { Population } \\
\end{array}$ & $\mathbf{N}$ & $\begin{array}{c}\text { Peak } \\
\text { Month }\end{array}$ & $\begin{array}{c}\text { Peak } \\
\text { Population } \\
\end{array}$ \\
\hline \multicolumn{10}{|l|}{ Questing Adults } \\
\hline \multicolumn{10}{|l|}{ Lyme disease risk } \\
\hline $\begin{array}{l}\text { Minimal vs. } \\
\text { Low/Medium/High }\end{array}$ & 461 & 0.82 & 0.81 & 214 & 0.68 & 0.67 & 1,008 & 0.60 & 0.64 \\
\hline $\begin{array}{l}\text { Minimal/Low vs. } \\
\text { Medium/High }\end{array}$ & 461 & 0.81 & 0.80 & 214 & 0.75 & 0.73 & 1,008 & 0.72 & 0.71 \\
\hline $\begin{array}{l}\text { Minimal/Low/Medium } \\
\text { vs. High }\end{array}$ & 461 & 0.89 & 0.92 & 214 & 0.72 & 0.71 & 1,008 & 0.70 & 0.70 \\
\hline Minimal vs. High & 226 & 0.95 & 0.96 & 88 & 0.79 & 0.78 & 530 & $\mathbf{0 . 7 7}$ & 0.78 \\
\hline \multicolumn{10}{|l|}{ Questing Nymphs } \\
\hline \multicolumn{10}{|l|}{ Lyme disease risk } \\
\hline $\begin{array}{l}\text { Minimal vs. } \\
\text { Low/Medium/High }\end{array}$ & 461 & 0.82 & 0.81 & 214 & 0.66 & 0.67 & 1,008 & 0.53 & 0.62 \\
\hline $\begin{array}{l}\text { Minimal/Low } v s \\
\text { Medium/High }\end{array}$ & 461 & 0.81 & 0.80 & 214 & 0.75 & 0.74 & 1,008 & 0.95 & 0.63 \\
\hline $\begin{array}{l}\text { Minimal/Low/Medium } \\
v s . \text { High }\end{array}$ & 461 & 0.91 & 0.91 & 214 & 0.72 & 0.72 & 1,008 & 0.94 & 0.62 \\
\hline Minimal vs. High & 226 & 0.96 & 0.96 & 88 & 0.78 & 0.78 & 530 & 0.97 & 0.78 \\
\hline \multicolumn{10}{|l|}{ Questing Larvae } \\
\hline \multicolumn{10}{|l|}{ Lyme disease risk } \\
\hline $\begin{array}{l}\text { Minimal } v s . \\
\text { Low/Medium/High }\end{array}$ & 461 & 0.82 & 0.81 & 214 & 0.66 & 0.63 & 1,008 & 0.53 & 0.62 \\
\hline $\begin{array}{l}\text { Minimal/Low vs. } \\
\text { Medium/High }\end{array}$ & 461 & 0.81 & 0.80 & 214 & 0.75 & 0.71 & 1,008 & 0.95 & 0.61 \\
\hline $\begin{array}{l}\text { Minimal/Low/Medium } \\
\text { vs. High }\end{array}$ & 461 & 0.91 & 0.90 & 214 & 0.72 & 0.71 & 1,008 & 0.94 & 0.58 \\
\hline Minimal vs. High & 226 & 0.96 & 0.96 & 88 & 0.78 & 0.76 & 530 & 0.97 & 0.63 \\
\hline
\end{tabular}

* Bold indicates significance; orange indicates AUC $>0.8$ and $p<0.05$; blue indicates $0.8>$ AUC $>0.7$ and $p<0.05$. 


\subsubsection{Spatial Sensitivity Analysis}

Regression coefficients produced in conditional autoregressive models for each DPF at each life stage on CDC data were not substantively different from those produced by a non-spatial regression (not shown). Conditional autoregressive models exhibited low values of $\tau^{2}(0.003,0.06)$ relative to the intercept and/or the parameter value, indicating that the contribution of spatial autocorrelation is small.

\subsection{Shifts in Geographic Distribution of DPFs}

DPFs in all cells $(\mathrm{N}=262,152)$ under future climate scenarios were significantly $(p<0.001)$ different than those from baseline simulations. Simulated mean, median and peak populations all show increases across most of the eastern United States, with the largest increases in the RCP 8.5 scenario. In particular, $18.8 \%, 7.7 \%$ and $4.1 \%$ of cells showed an increase of an order of magnitude or greater in peak QA, QN and QL population, respectively, in the RCP 8.5 scenario. Relative to baseline, regions of highest projected mean, median and peak tick population expanded both northward and southward to encroach upon the areas of low DPF values occurring across the Appalachian mountain range (e.g., Figure 1).

While both projected scenarios showed simulated questing life stage average Peaks per Year that were significantly different from the baseline case and the two projected scenarios were significantly different from one another, there was no substantive change $(<0.01$ peaks per year) when these comparisons were made across the entire domain. Variations in population response between scenarios, as shown by Peaks per Year, demonstrate the lack of uniform response (Figure 1). The number of cells projected to have two or more population peaks per year for the QA life stage increased in northern regions, while there was a net decrease in the number of cells with more than one peak in southern regions. In contrast, for the QL and QN life stages, the number of cells experiencing more than one population peak per year decreased uniformly across the spatial domain.

Under scenarios RCP 4.5 and RCP 8.5 compared to baseline, the season length for QA, as defined by $I P$ to IP, has a pronounced increase from 50-70 day seasons to 60-90 and 80-100 day seasons, respectively, in the South. In the North, there is a net decrease in QA season length for both future scenarios (Figure 1). For QN, projected season length remained the same as baseline, though there was a slight decrease in season length for much of the northern portion of the study area in both projected scenarios, and an increase of approximately 40 days in season length in a small portion of the land-locked Midwest. Projected QL season length showed a decrease of approximately 10 days in southern areas, while the overall geographic area with higher season lengths decreased with increasing scenario severity. Changes in projected season length, as defined by Peak to Trough, were approximately uniform across the domain (not shown). QA Peak to Trough lengthened on average by eight days in RCP 4.5, and by 1.4 days in RCP 8.5, as compared to baseline. QN and QL Peak to Trough shortened by 56.6 and 31.1 days, respectively, in the RCP 8.5 scenario, and by 15 and 10 days, respectively, in the RCP 4.5 scenario. 
Figure 1. (A) Log of Peak Population, (B) IP to IP, (C) log of IP Pop, (D) Peak Month, (E) $U Q / I Q R$ and (F) Peak to Trough for questing adults (QA), questing nymphs (QN) and questing larvae (QL).
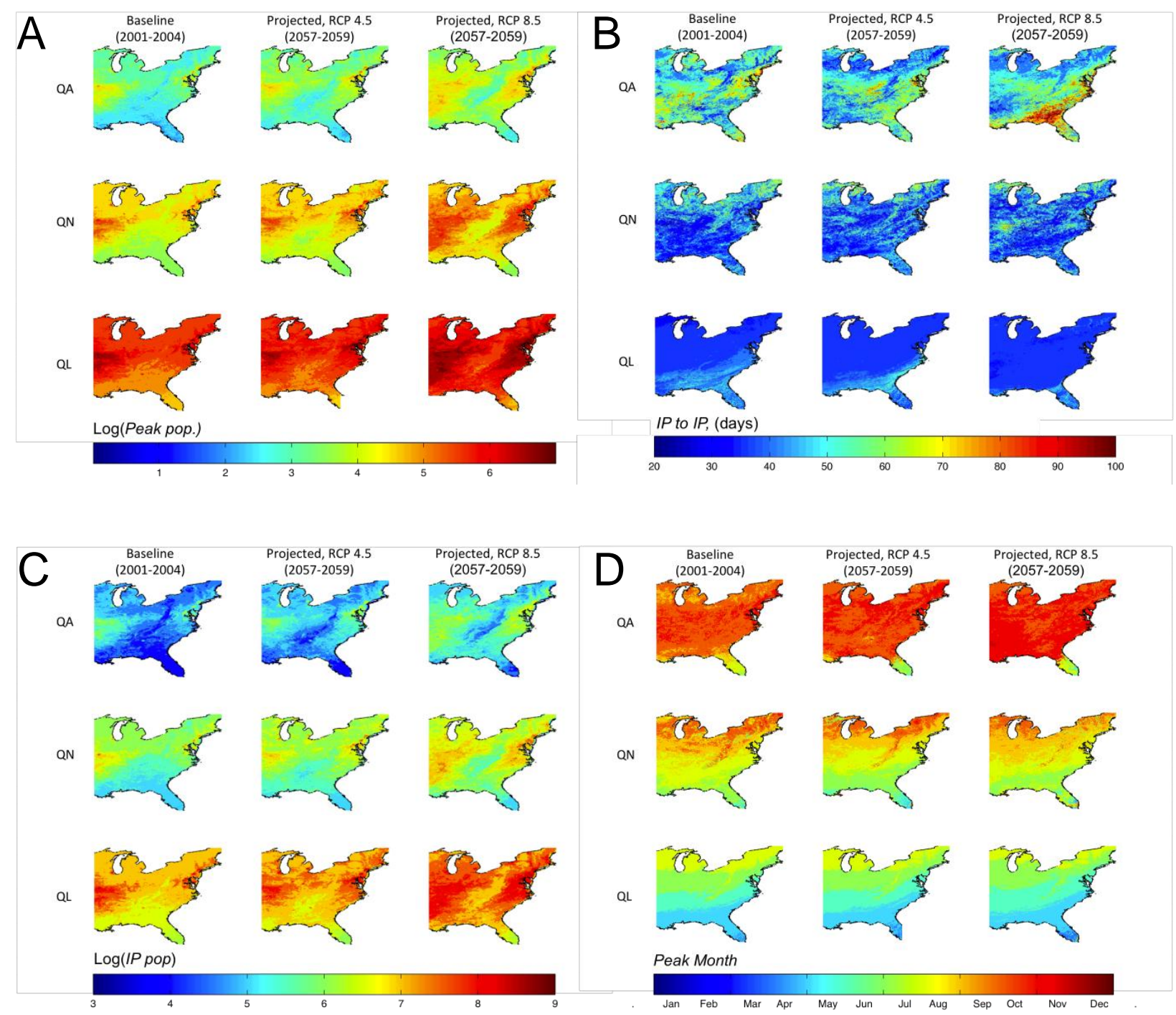

QN
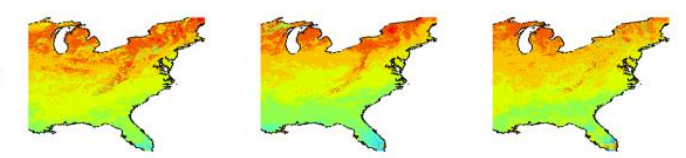

QL
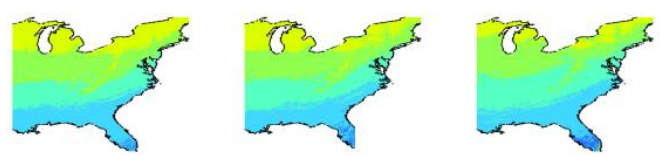

Peak Month
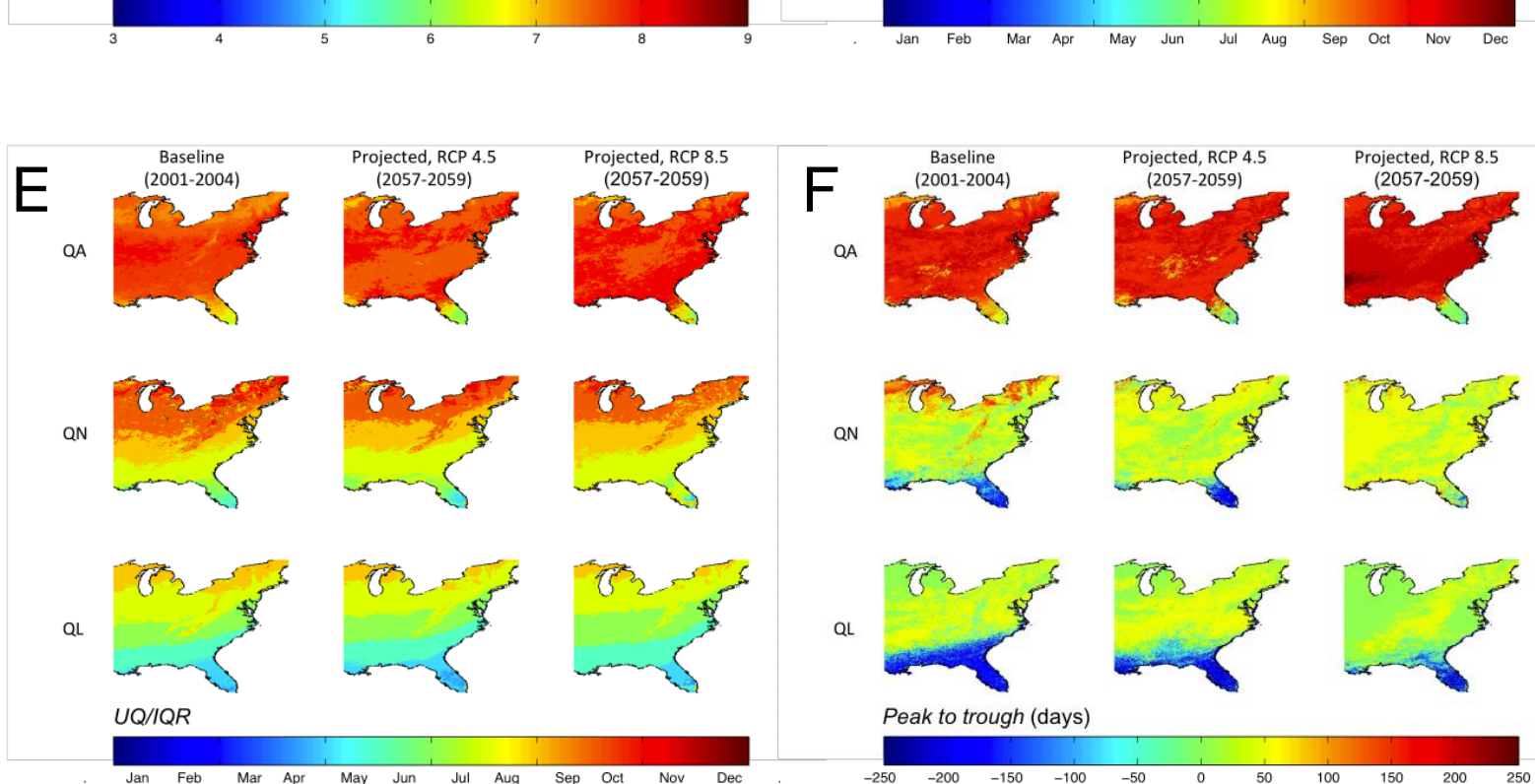

QL
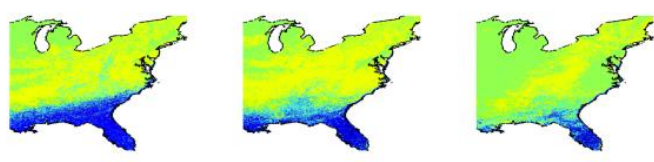

Peak to trough (days) 
The exposure DPF, IP Pop, which counts the number of tick-days during a season bounded by inflection points around the yearly maximum population, bears little similarity to IP to IP maps (Figure 1). IP Pop increases in exposure-time across the domain as the severity of the projected scenarios increases. The regions with the highest baseline number of ticks, present during the inflection point defined season, spread outward in both the north and south direction and center around the Midwest and the Northeast.

Wave Angle results (see Supplementary Information; Figure S1) showed that the dynamics of all life stages under both projected climate conditions lag behind those at baseline climate by $\leq 4$ days across the simulated domain. The projected month of peak population (Peak Month) and UQ/IQR for QA generally shifted to earlier months. However, with increasing scenario severity, QN and QL generally shifted to later months across the geographic area.

Wave angle results (see Figure S1) showed that the dynamics of all life stages under both projected climate conditions lag behind those at baseline climate by $\leq 4$ days across the simulated domain. The projected month of peak population (Peak Month) and UQ/IQR for QA generally shifted to earlier months. However, with increasing scenario severity, QN and QL generally shifted to later months across the geographic area.

\section{Discussion and Conclusions}

When examining the response of vector populations to climate change, shifts in phenology, seasonality and other dynamic characteristics can be anticipated across the spatial range and life stages of the organism of interest. Risk of VBD is dependent on both timing and probability of exposure to the vector, and thus, characterizing the dynamic population response over space is crucial in order to anticipate and manage potential future risks. Here, we provide a framework for evaluating both static and dynamic effects of climate change on populations over large geographic areas, using spatially explicit simulation of a climate-driven, stage-structured population model.

Our findings with respect to Ixodes scapularis illustrate both the methodology and its utility. The derivation and analysis of dynamic population features are key to the analytical approach. DPFs provide quantitative information about a range of population characteristics and allow for comparison between dynamic simulation output and observed disease data, as well as between baseline and projected climates. Absolute DPFs, such as Mean, Median and Peak Population can be interpreted as indicators of survivorship, while timing DPFs, such as number of days from the yearly maximum population to the yearly minimum population (Peak to Trough) and month in which peak population occurs (Peak Month) characterize the timing and length of a given life stage's season.

In the case of Ixodes, DPFs associated with the peak of the simulated population curve, Peak Population and Peak Month, proved to be the most important in predicting high risk of Lyme disease, though all DPFs showed some level of discriminatory ability. AUC analyses showed that dichotomizations isolating high risk improved discriminatory ability across all DPFs and life stages. Aggregation of medium and high risk also showed improved discriminatory ability across life stages and DPFs as compared to the minimal vs. low/medium/high dichotomization. This trend of improvement, as high disease risk is progressively isolated into a single category, suggests strongly that these DPFs are useful in predicting the timing and location of higher Lyme disease risks. 
When DPFs are examined for two projected climate scenarios, we show that the dynamic population response of I. scapularis is not uniform across life stages and varies over space. Spatial shifts in temporal features include geographic shifts in season, and these shifts are not consistently northward as one might intuitively hypothesize. While the month in which the greatest number of ticks are questing (Peak Month) is delayed for the adult life stage (Figure 1), QN and QL peaks do not show geographically uniform shifts to earlier questing season. Also, Peak to Trough and IP to IP indicate potential changes in season length in projected scenarios. Spatial shifts in absolute DPFs, such as Peak Population, vary by region. For instance, the peak populations in the Midwest and the Northeast regions are both expected to rise far more as compared to the Appalachian mountain range or the Gulf Coast, where these populations are expected to remain more stable.

Although the finding that QL Peak Month and Peak Population show high predictive ability for Lyme disease risk is significant, the causal implications of this finding, and others like it, must be interpreted cautiously. Disease risk is not directly related to the questing larval stage, which takes the first blood meal in the lifecycle, and thus, is responsible for Lyme transmission only under the rare circumstance that larvae are infected transovarially. Likewise, QN Peak Month and Peak Population have similar AUC values for all dichotomizations of Lyme disease risk, an effect driven largely by the similarity of tick response to temperature in these two life stages, rather than mutual causal relationships with disease. Complex temporal relationships are inherent in these populations: questing nymphs and questing larvae, for instance, peak at approximately the same time of year, and their populations in a given location are ostensibly correlated, though the QN population does not result from the QL population in the same year, but rather previous years' QL.

As in other ecological modeling analyses, data quality determines the utility of this analysis framework for a given system. In our analysis, CDC data quality may account for the lack of significant AUCs of DPFs in comparison to the observed tick data. Tick presence/absence data are collected using a variety of methods, such as dragging and deer surveys, often under serious resource constraints [33]. Rather than providing consistent, systematic information about tick presence and absence, the national tick dataset offers a coarse categorization derived from disparate information. This is in contrast to the national Lyme disease dataset, which is based on a consistent reporting standard. Given the higher quality of data collected, this dataset is more useful in substantiating the results of our model.

Other climate factors besides temperature, such as humidity, have been shown to affect Ixodes spp. activity [41,42] and correlate with human Lyme disease risk [43]. The population model used here did not incorporate Ixodes' response to humidity, and although our simulated population data demonstrated good correspondence with Lyme incidence, it is possible that including other key environmental variables may yield yet greater correspondence. Likewise, host and pathogen populations were not considered in our analysis, which was limited to vector dynamics. Relatively little research has been done on the potential population responses of Borrelia spp. under altered climate conditions. However, it has been suggested that changes in Ixodes phenology in response to climatic changes may affect the evolution of various tick-borne pathogens, so as to modify their lifespan, transmission and pathogenicity [44]. Host dynamics can also greatly impact infected vector density and consequent human risk in a variety of VBD systems [45-47]. In the case of Lyme disease, the abundance of key hosts, such as mice and chipmunks, has been shown to predict the density of 
infected nymphs in eastern deciduous forests [48]; in other areas, such as the southern United States, lizards are believed to exert a dampening effect on the spread of Lyme disease, due to poor host competence or zooprophylactic effects [49,50]. Including host, vector and pathogen dynamics in a combined model would pose significant methodological and computational challenges, but is also likely to add greatly to our mechanistic understanding of shifting VBD risk under future environmental conditions. We note that a similar simulation, summarization (e.g., DPFs) and analysis approach can be pursued with such a combined model; yet, other summarizations (e.g., $R_{0}$ ) become available for geovisualization in that context (e.g., [51,52]).

The methodological contributions made by the modeling analysis described here are considerable. We provide a quantitative assessment of population dynamics - with potential consequences for disease risk - under future climates, which is made possible by use of a spatially-explicit, mechanistic model [53]. Our spatial characterization of DPFs allows a detailed visual assessment (e.g., Figure 1), alongside a quantitative analysis, of the dynamic population response to future climate, revealing potential changes that are non-intuitive. For instance, across the eastern United States, under projected temperatures as compared to the baseline scenario, nymphs and larvae are projected to arrive at their peak population earlier in the season, while adults are projected to reach peak population later in the season (Figure 1). The approach taken here also highlights the value of modeling abundance, which, unlike habitat suitability or other static measures, allows for the examination of phenology and seasonality among life stages and the potential implications for (and correlation with) disease risk. For instance, IP to IP indicates that the length of larval "season" is stable across the three temperature scenarios, while the adult, and, to a lesser extent, the nymphal stages exhibit "seasons" that are strongly sensitive to the projected increasing temperatures. Such life-stage-specific responses in time and space would be unapparent using traditional methods that examine, for instance, aggregate, annual effects.

We caution above against a causal interpretation of a DPF's predictive power. A strong correlation between a DPF and observed disease incidence may not represent a causal relationship, but such a finding can raise hypotheses that ultimately lead to greater mechanistic understanding of the relationship between vector populations and disease risk in space and time and, thus, an improved causal understanding. Finally, population models, such as the one examined here, can also be used to evaluate the efficacy and economy of potential public health interventions [53], such as vector or host control (e.g., [54-56] for Lyme disease). A coupled analysis of the effect of temperature in the presence of a vector control program would be an obvious extension of the approach, and such an application of this model is possible for many different vectors, interventions and diseases.

We have demonstrated the ability of a spatially-explicit dynamic population model to discriminate between dynamic population features most strongly associated with disease risk, as well as to characterize the geographically varied response of I. scapularis life stages to climate dynamics. Use of such an approach to describe shifts in dynamics is not limited to Lyme disease. The technique may provide new insights into the dynamic responses of a range of disease vectors to environmental changes, particularly shifts in their seasonal and phenological features. Such analyses may provide helpful information about the consequent risk of vector-borne disease under future conditions. 


\section{Acknowledgments}

Thanks to R. Patrick Mayne, Julia Moore, Jess Belle, Jianyong Wu and the rest of the Remais Lab. This work was supported in part by the CDC Climate and Health Program (award \# 5 U01 EH000405), the Ecology of Infectious Disease program of the National Science Foundation under Grant No. 0622743, the National Institute for Allergy and Infectious Disease (K01AI091864), the Global Health Institute at Emory University and the National Science Foundation through TeraGrid (now XSEDE). Resources for climate simulation data conducted at UT/ORNL were provided by the National Institute for Computational Sciences (NICS) under grant number TG-ATM110009. The funders had no role in study design, data collection and analysis, decision to publish or preparation of the manuscript. We acknowledge the support of George Luber, who leads the CDC Climate and Health Program.

\section{Conflict of Interest}

The authors declare no conflict of interest.

\section{References}

1. Dobson, A. Climate variability, global change, immunity, and the dynamics of infectious diseases. Ecology 2009, 90, 920-927.

2. Altizer, S.; Dobson, A.; Hosseini, P.; Hudson, P.; Pascual, M.; Rohani, P. Seasonality and the dynamics of infectious diseases. Ecol. Lett. 2006, 9, 467-484.

3. Kutz, S.J.; Hoberg, E.P.; Polley, L.; Jenkins, E.J. Global warming is changing the dynamics of Arctic host-parasite systems. Proc. Roy. Soc. B-Biol. Sci. 2005, 272, 2571-2576.

4. Lafferty, K.D. The ecology of climate change and infectious diseases. Ecology 2009, 90, 888-900.

5. Confalonieri, U.; Menne, B.; Akhtar, R.; Ebi, K.L.; Hauengue, M.; Kovats, R.S.; Revich, B.; Woodward, A. Human Health. In Climate Change 2007: Impacts, Adaptation and Vulnerability; Parry, M.L., Canziani, O.F., Palutikof, J.P., van der Linden, P.J., Hanson, C.E., Eds.; Cambridge University Press: Cambridge, UK, 2007; pp. 391-431.

6. Ostfeld, R.S. Climate change and the distribution and intensity of infectious diseases. Ecology 2009, 90, 903-905.

7. Pascual, M.; Bouma, M.J. Do rising temperatures matter? Ecology 2009, 90, 906-912.

8. Randolph, S.E. Perspectives on climate change impacts on infectious diseases. Ecology 2009, 90, 927-931.

9. Svoray, T.; Shafran-Nathan, R.; Henkin, Z.; Perevolotsky, A. Spatially and temporally explicit modeling of conditions for primary production of annuals in dry environments. Ecol. Model. 2008, 218, 339-353.

10. Bentz, B.J.; Regniere, J.; Fettig, C.J.; Hansen, E.M.; Hayes, J.L.; Hicke, J.A.; Kelsey, R.G.; Negron, J.F.; Seybold, S.J. Climate change and bark beetles of the western United States and Canada: Direct and indirect effects. Bioscience 2010, 60, 602-613.

11. Corson, M.S.; Teel, P.D.; Grant, W.E. Microclimate influence in a physiological model of cattle-fever tick (Boophilus spp.) population dynamics. Ecol. Model. 2004, 180, 487-514. 
12. White, N.; Sutherst, R.W.; Hall, N.; Whish-Wilson, P. The vulnerability of the Australian beef industry to impacts of the cattle tick (Boophilus microplus) under climate change. Climatic Change 2003, 61, 157-190.

13. Erickson, R.A.; Presley, S.M.; Allen, L.J.S.; Long, K.R.; Cox, S.B. A dengue model with a dynamic Aedes albopictus vector population. Ecol. Model. 2010, 221, 2899-2908.

14. Jacobson, A.R.; Provenzale, A.; von Hardenberg, A.; Bassano, B.; Festa-Bianchet, M. Climate forcing and density dependence in a mountain ungulate population. Ecology 2004, 85, 1598-1610.

15. Mount, G.A.; Haile, D.G.; Daniels, E. Simulation of blacklegged tick (Acari: Ixodidae) population dynamics and transmission of Borrelia burgdorferi. J. Med. Entomol. 1997, 34, 461-484.

16. Sauvage, F.; Langlais, M.; Pontier, D. Predicting the emergence of human hantavirus disease using a combination of viral dynamics and rodent demographic patterns. Epidemiol. Infect. 2007, 135, 46-56.

17. Brownstein, J.S.; Holford, T.R.; Fish, D. Effect of climate change on Lyme disease risk in North America. EcoHealth 2005, 2, 38-46.

18. Diuk-Wasser, M.A.; Vourc'h, G.; Cislo, P.; Hoen, A.G.; Melton, F.; Hamer, S.A.; Rowland, M.; Cortinas, R.; Hickling, G.J.; Tsao, J.I. Field and climate-based model for predicting the density of host-seeking nymphal Ixodes scapularis, an important vector of tick-borne disease agents in the eastern United States. Global. Ecol. Biogeogr. 2010, 19, 504-514.

19. Chuine, I. Why does phenology drive species distribution? Phil. Trans. Biol. Sci. 2010, 365, 3149-3160.

20. Visser, M.E.; Both, C. Shifts in phenology due to global climate change: The need for a yardstick. Proc. Roy. Soc. B-Biol. Sci. 2005, 272, 2561-2569.

21. Austin, M.P. Spatial prediction of species distribution: an interface between ecological theory and statistical modelling. Ecol. Model. 2002, 157, 101-118.

22. Guisan, A.; Thuiller, W. Predicting species distribution: Offering more than simple habitat models. Ecol. Lett. 2005, 8, 993-1009.

23. Chuine, I.; Beaubien, E.G. Phenology is a major determinant of tree species range. Ecol. Lett. 2001, 4, 500-510.

24. Pitt, J.P.W.; Regniere, J.; Worner, S. Risk assessment of the gypsy moth, Lymantria dispar (L), in New Zealand based on phenology modelling. Int. J. Biometeorol. 2007, 51, 295-305.

25. Killilea, M.E.; Swei, A.; Lane, R.S.; Briggs, C.J.; Ostfeld, R.S. Spatial dynamics of Lyme disease: A review. EcoHealth 2008, 5, 167-195.

26. Tonnang, H.E.Z.; Kangalawe, R.Y.M.; Yanda, P.Z. Predicting and mapping malaria under climate change scenarios: The potential redistribution of malaria vectors in Africa. Malar. J. 2010, 9 , doi: 10.1186/1475-2875-9-111.

27. Ebi, K.L.; Hartman, J.; Chan, N.; McConnell, J.; Schlesinger, M.; Weyant, J. Climate suitability for stable malaria transmission in Zimbabwe under different climate change scenarios. Climatic Change 2005, 73, 375-393.

28. Ogden, N.H.; Bigras-Poulin, M.; O’Callaghan, C.J.; Barker, I.K.; Lindsay, L.R.; Maarouf, A.; Smoyer-Tomic, K.E.; Waltner-Toews, D.; Charron, D. A dynamic population model to investigate effects of climate on geographic range and seasonality of the tick Ixodes scapularis. Int. J. Parasitol. 2005, 35, 375-389. 
29. Zhou, X.N.; Yang, G.J.; Yang, K.; Wang, X.H.; Hong, Q.B.; Sun, L.P.; Malone, J.B.; Kristensen, T.K.; Bergquist, N.R.; Utzinger, J. Potential impact of climate change on schistosomiasis transmission in China. Amer. J. Trop. Med. Hyg. 2008, 78, 188-194.

30. Moore, J.; Liang, S.; Akullian, A.; Remais, J. Cautioning the use of degree-day models for climate change projections: Predicting the future distribution of parasite hosts in the presence of parametric uncertainty. Ecol. Appl. 2012, 22, 2237-2247.

31. Hales, S.; de Wet, N.; Maindonald, J.; Woodward, A. Potential effect of population and climate changes on global distribution of dengue fever: An empirical model. Lancet 2002, 360, 830-834.

32. Ogden, N.H.; Maarouf, A.; Barker, I.K.; Bigras-Poulin, M.; Lindsay, L.R.; Morshed, M.G.; O'Callaghan, C.J.; Ramay, F.; Waltner-Toews, D.; Charron, D.F. Climate change and the potential for range expansion of the Lyme disease vector Ixodes scapularis in Canada. Int. J. Parasitol. 2006, 36, 63-70.

33. Dennis, D.T.; Nekomoto, T.S.; Victor, J.C.; Paul, W.S.; Piesman, J. Reported distribution of Ixodes scapularis and Ixodes pacificus (Acari: Ixodidae) in the United States. J. Med. Entomol. 1998, 35, 629-638.

34. Goddard, J. Ecological studies of Ixodes scapularis (Acari: Ixodidae) in central Mississippi: Lateral movement of adult ticks. J. Med. Entomol. 1993, 30, 824-826.

35. Lane, R.S.; Mun, J.; Stubbs, H.A. Horizontal and vertical movements of host-seeking Ixodes pacificus (Acari: Ixodidae) nymphs in a hardwood forest. J. Vector Ecol. 2009, 34, 252-266.

36. Gao, Y.; Fu, J.S.; Drake, J.B.; Liu, Y.; Lamarque, J.F. Projected changes of extreme weather events in the eastern United States based on a high resolution climate modeling system. Environ. Res. Lett. 2012, 7; 1-12.

37. Goosse, H.; Barriat, P.Y.; Lefebvre, W.; Loutre, M.F.; Zunz, V. Chapter 6. Future Climate Changes. In Introduction to Climate Dynamics and Climate Modeling. Available online: http://www.climate.be/textbook (accessed on 1 March 2012).

38. Grenfell, B.T.; Bjornstad, O.N.; Kappey, J. Travelling waves and spatial hierarchies in measles epidemics. Nature 2001, 414, 716-723.

39. Centers for Disease Control and Prevention (CDC). Recommendations for the Use of Lyme Disease Vaccine: Recommendations of the Advisory Committee on Immunization Practices; CDC: Atlanta, GA, USA, 1999; p. 21.

40. Lee, D. A comparison of conditional autoregressive models used in Bayesian disease mapping. Spat. Spatio-Temporal Epidemiol. 2011, 2, 78-89.

41. Perret, J.-L.; Guigoz, E.; Rais, O.; Gern, L. Influence of saturation deficit and temperature on Ixodes ricinus tick questing activity in a Lyme borreliosis-endemic area (Switzerland). Parasitol. Res. 2000, 86, 554-557.

42. Vail, S.G.; Smith, G. Air temperature and relative humidity effects on behavioral activity of blacklegged tick (Acari: Ixodidae) nymphs in New Jersey. J. Med. Entomol. 1998, 35, 1025-1028.

43. Bennet, L.; Halling, A.; Berglund, J. Increased incidence of Lyme borreliosis in Southern Sweden following mild winters and during warm, humid summers. Eur. J. Clin. Microbiol. Infect. D. 2006, 25, 426-432. 
44. Ogden, N.H.; Bigras-Poulin, M.; Hanincova, K.; Maarouf, A.; O’Callaghan, C.J.; Kurtenbach, K. Projected effects of climate change on tick phenology and fitness of pathogens transmitted by the North American tick Ixodes scapularis. J. Theor. Biol. 2008, 254, 621-632.

45. Adler, F.R.; Pearce-Duvet, J.M.C.; Dearing, M.D. How host population dynamics translate into time-lagged prevalence: An investigation of Sin Nombre virus in deer mice. Bull. Math. Biol. 2008, 70, 236-252.

46. Dobson, A. Population dynamics of pathogens with multiple host species. Amer. Naturalist 2004, 164, S64-S78.

47. Kiffner, C.; Zucchini, W.; Schomaker, P.; Vor, T.; Hagedorn, P.; Niedrig, M.; Rühe, F. Determinants of tick-borne encephalitis in counties of southern Germany, 2001-2008. Int. J. Health Geogr. 2010, 9, 42-42.

48. Ostfeld, R.S.; Canham, C.D.; Oggenfuss, K.; Winchcombe, R.J.; Keesing, F. Climate, deer, rodents, and acorns as determinants of variation in Lyme-Disease risk. Plos. Biol. 2006, 4, doi: 10.1371/journal.pbio.0040145.

49. Lane, R.S.; Quistad, G.B. Borreliacidal factor in the blood of the western Fence Lizard (Sceloporus occidentalis). J. Parasitol. 1998, 84, 29-34.

50. Oliver, J.H.; Cummins, G.A.; Joiner, M.S. Immature Ixodes scapularis (Acari: Ixodidae) parasitizing lizards from the southeastern USA. J. Parasitol. 1993, 79, 684-689.

51. Gething, P.; Patil, A.; Smith, D.; Guerra, C.; Elyazar, I.; Johnston, G.; Tatem, A.; Hay, S. A new world malaria map: Plasmodium falciparum endemicity in 2010. Malar. J. 2011, 10, doi: 10.1186/1475-2875-10-378.

52. Boender, G.J.; van Roermund, H.J.; de Jong, M.C.; Hagenaars, T.J. Transmission risks and control of foot-and-mouth disease in The Netherlands: spatial patterns. Epidemics 2010, 2, 36-47.

53. Rogers, D.; Randolph, S. Climate change and vector-borne diseases. Advan. Parasitol. 2006, 62, 345-381.

54. Daniels, T.J.; Falco, R.C.; McHugh, E.E.; Vellozzi, J.; Boccia, T.; Denicola, A.J.; Pound, J.M.; Miller, J.A.; George, J.E.; Fish, D. Acaricidal treatment of white-tailed deer to control Ixodes scapularis (Acari: Ixodidae) in a New York Lyme disease-endemic community. Vector-Borne Zoonotic Dis. 2009, 9, 381-387.

55. Garnett, J.M.; Connally, N.P.; Stafford, K.C., III; Cartter, M.L. Evaluation of deer-targeted interventions on Lyme disease incidence in Connecticut. Public Health Rep. 2011, 126, 446-454.

56. Hayes, E.B.; Piesman, J. How can we prevent Lyme disease? N. Engl. J. Med. 2003, 348, 2424-2430.

(C) 2013 by the authors; licensee MDPI, Basel, Switzerland. This article is an open access article distributed under the terms and conditions of the Creative Commons Attribution license (http://creativecommons.org/licenses/by/3.0/). 